\title{
Periarthritis shoulder - Effect of manipulation under general anesthesia on pain and range of movement
}

\author{
Sandeep Krishna Avulapati \\ Assistant Professor, Dept. of Orthopedics, BIRRD (T) Hospital, Sri Padmavathi Medical College for Women, SVIMS University, \\ Tirupati, Andhra Pradesh, India \\ *Corresponding Author: Sandeep Krishna Avulapati \\ Email: sundeepavulapati@gmail.com
}

\begin{abstract}
Introduction: Periarthritis shoulder is unique condition, in sense that same pathology does not affect any other joints in particular other than shoulder. ${ }^{2}$ Spontaneous occurrence of pain in shoulder unrelated to injury, but at times associated with injury in $20-30 \%$ of cases. $^{2}$ Main aim of treatment is symptomatic pain relief followed by restoration of normal joint movements. Manipulation under General Anesthesia (MUA) appears to be mainstay of treatment for people who do not benefit by physiotherapy and universal shoulder mobilization exercises. ${ }^{3}$

Aim of Study: To study effect of manipulation under general anesthesia on Periarthritis shoulder.

Objective: To study improvement in Range of Movements, Pain and Short DASH score.

Materials and Methods: Study was carried out in Pushpagiri Institute of Medical Sciences and Research Centre during September 2010 to September 2012. 40 patients were selected for the study, underwent MUA with intra-articular steroid injection $(40 \mathrm{mg} / \mathrm{ml}$ methyl prednisolone), $2 \%$ Lignocaine $(5 \mathrm{ml})$ and Hydrodilation $(40 \mathrm{ml}$ cold normal saline). They were started physiotherapy immediately and continued.

Result: Among the 40 patients 6 patients never returned for follow-up. Data analysis of remaining 34 patients shows improvement in shoulder range of movement. Quick DASH score decreased from 83.72(S.D + 7.1) to 27.66 (S.D + 7.0). VAS score improved from 7.29 (S.D + 1.03) to 1.71 (S.D + 0.84).

Conclusion: Periarthritis shoulder, treated with MUA with intra-articular steroid (steroid+Lignocaine) and hydrodilation (with normal saline) result in good immediate pain relief postoperatively and easy breakage of adhesions during the procedure. This followed by immediate physiotherapy decreases pain, adds good functional recovery and early return to daily activities of living. Physiotherapy and universal shoulder mobilization exercises should be continued thereafter to prevent recurrence of symptoms.
\end{abstract}

Keywords: Frozen shoulder syndrome, Adhesive capsulitis, Periarthritis, Manipulation under anaesthesia, Corticosteroids, rehabilitation, Shoulder.

\section{Introduction}

Periarthritis shoulder is a disorder characterized by progressive pain and stiffness of the shoulder which usually resolves spontaneously after about 18 months. ${ }^{4}$ Diabetes $^{5}$ and hemiplegia ${ }^{6}$ increase the risk of this condition, but in most cases no predisposing disorders are noted. Pain and contracture are the two principal characteristic features. Shoulder pain is the third common cause of musculoskeletal disability after low back pain and neck pain. ${ }^{4}$ It is the multidirectional limitation of passive movements of more than $50 \%$ in the gleno-humeral joint in abduction, forward flexion and external rotation. NSAIDS, physiotherapy, universal shoulder mobilization exercises, manipulation under General Anesthesia, intra-articular steroid injections, hydrodilation with cold normal saline, arthroscopic release and further surgical release are various methods of treatment for Periarthritis.

Further in Literature, there are certain conflicting views over the

1. Outcome of steroid usuage,,$^{13}$

2. Physiotherapy alone and MUA combined with physiotherapy showed similar results with long term follow-up. ${ }^{12}$

3. Hydrodilation better than Manipulation alone. ${ }^{14}$
4. MUA followed by immediate physiotherapy showed better results. ${ }^{9,15}$

Our aim of the study is to evaluate effect of MUA of Periarthritis shoulder. This includes observation of effect of intra-articular steroid injection (steroid + Lignocaine) and hydrodilation with cold normal saline during and after the procedure in terms of pain relief. Role of physiotherapy and universal shoulder mobilization exercises thereafter, for further improvement in range of movement and early return of daily activities of living in the individuals.

\section{Materials and Methods}

40 patients with Periarthritis shoulder who were admitted in the department of Orthopaedics, Pushpagiri Medical College Hospital, Thiruvalla, Kerala, from September, 2010 to September, 2012 were selected for the study.

\section{Inclusion Criteria}

1. Periarthritis shoulder should be characterized by restricted active and passive combined movements (scapulothoracic and glenohumeral) for more than a month duration which has either reached a plateau or worsened.

2. Persons who have undergone more than a month of conservative methods and have shown no 
improvement, including NSAIDS or steroids (orally), physiotherapy or universal shoulder mobilization exercises.

3. Patients with oxford shoulder score less than 19.

\section{Exclusion Criteria}

1. Glenohumeral arthritis

2. Any previous shoulder surgery

3. Previous history of shoulder dislocation and fracture dislocation

4. Diabetes.

5. Psychological problems e.g. dementia

6. Malignancy

Periarthritis shoulder diagnosis was based on history of pain and restricted active and passive glenohumeral joint movement in absence of any other secondary conditions like trauma etc which were excluded.

\section{Local Examination of Shoulder}

Passive mobility in flexion, abduction and external rotation was measured by using goniometer.

Mobility in flexion and abduction was defined by the maximal angle between the arm and midline of the body while the subject was in an erect position. Mobility in external rotation was defined by the maximal angle between the vertical line and the ante brachium when the patient was supine and his elbow was flexed 90. Mobility of inner rotation was graded from 1 to 15 , according to the patient ability to bring his hand behind his back. Hand at hip level was graded as 1, hand at level of vertebra L4 as 7 and hand at the level of vertebra $\mathrm{T} 5$ as 15 .

Investigations: Antero-posterior X-rays were taken to exclude other shoulder disorders. Routine investigations required for pre-anesthetic check-up (required for fitness for surgery) were carried out. Patients were informed about the possible effects of manipulation and corticosteroids. Pre operatively shoulder ROM, VAS Score charting and Quick DASH score were estimated.

\section{Preoperative Preparation of Patients}

1. Patients were kept fasting for 6 hours before surgery.

2. A written informed consent for procedure was taken.

3. The neck, chest, axilla shoulders and arm were prepared.

4. Tranquilizers were given as advised by the anesthetist.

Manipulation under anesthesia was performed by relaxation using general anesthesia administered by the anesthesiologist.

Procedure: Under aseptic precautions all patients are given $40 \mathrm{mg} / \mathrm{ml}$ methyl prednisolone intra-articularly. All the patients are given $5 \mathrm{cc} 2 \%$ lignocaine injection intra-articularly for post operative pain relief. Day before the procedure $100 \mathrm{ml}$ of normal saline will stored in refrigerator. All the patients are given $40 \mathrm{cc}$ cold normal saline intra-articularly before the procedure. Manipulation (in accordance with Codman's Paradox described by Codman in 1934), as follows. Elevation of the arm in the plane of the scapula while stabilizing the scapula with very short lever arm. Bringing the arm that is in full external rotation down by the side without any rotation, thus, tearing the anterior capsule. Adducting the arm across the body to extend the tear to the posterior capsule. Holding the arm in internal rotation and cross adducting without any rotation to complete the tear of the posterior-superior capsule. This procedure was followed for safe manipulation of shoulder.

Post-Operatively: Adequate post-operative analgesics, rest, icepack were given. Patients are started physiotherapy and active shoulder mobilization exercises on the same day. On $1^{\text {st }}$ Post-operative day Shoulder mobilization and physiotherapy continued. Shoulder ROM is estimated. Physiotherapy will be continued for a week which includes TENS, Ultrasound and Universal shoulder mobilization exercises. Later patients are followed regularly as OPD patients. They are asked whether they are continuing shoulder mobilization exercises or not. At the end of $4^{\text {th }}$ month shoulder ROM, VAS score and Quick DASH score were estimated

\section{Statistical Analysis}

Data was analyzed with paired Student $\mathrm{T}$ Test. For all statistical evaluations probability value $(\mathrm{P})=0.0005$ was considered significant.

\section{Observations and Results}

Complete data was available for 34 patients $(85 \%)$, remaining 6 patients did not turn-up for follow-up, hence excluded from the study. Average age of subjects is 52 years, with Range (39-65 years). Of 34, 18 are male and 16 are female patients. 18 patients had right shoulder and remaining left shoulder. All 34 patients achieved significant reduction in pain immediately after manipulation which is due to $5 \mathrm{ml} 2 \%$ Lignocaine injections intra-articularly. VAS score before MUA was 7.29 (S.D \pm 1.03$)$ and reduced to $1.71(\mathrm{~S} . \mathrm{D} \pm 0.84)$ at the end of 4 months. The Quick DASH score, represents daily activities of living involving the shoulder also showed significant improvement from 83.7 (S.D + 7.15) to $27.6(\mathrm{~S} . \mathrm{D} \pm 7.04)$.

There was significant improvement in all ROM following manipulation. Flexion improved from $102.52^{0}\left(\mathrm{~S} . \mathrm{D} \pm 7.9^{0}\right)$ to $157.52^{0}\left(\mathrm{~S} . \mathrm{D} \pm 5.5^{\circ}\right)$, abduction from $82.85^{0}\left(\mathrm{~S} . \mathrm{D} \pm 7.1^{0}\right)$ to $150.76^{0}\left(\mathrm{~S} . \mathrm{D} \pm 3.9^{0}\right)$, external rotation from $27.17^{0}$ S.D $\pm 3.8^{0)}$ to $45.44^{0}$ (S.D $\left.\pm 4^{0}\right)$, internal rotation from grade $2.52(\mathrm{~S} . \mathrm{D} \pm 1)$ to 7.5 (S.D \pm 1$)$. No intra-operative complication was encountered during the MUA in patients. In particular there was no glenohumeral dislocation or fractures of humerus. All the patients received physiotherapy immediately after MUA. They all continued universal 
shoulder mobilization exercises. No significant difference between male and female patients in VAS score, ROM shoulder and Quick DASH score was observed on statistical analysis.

Table 1: Mean VAS Score before and after the manipulation of shoulder

\begin{tabular}{|l|c|c|}
\hline \multicolumn{1}{|c|}{ Condition } & VAS Score Mean & Standard deviation \\
\hline Before the Manipulation of Shoulder & 7.29 & 1.03 \\
\hline After the Manipulation of Shoulder & 1.71 & 0.84 \\
\hline
\end{tabular}

$\mathrm{P}$ value $=0.0005$

Table 2: Comparison of ROM shoulder measured on three occasions; 1 . Before the manipulation; $2.1^{\text {st }}$ day after the manipulation; 3. 4 months after the manipulation

\begin{tabular}{|l|c|c|c|}
\hline Movement of shoulder & Before the Manipulation & $\begin{array}{c}\mathbf{1}^{\text {st }} \text { day After the } \\
\text { manipulation }\end{array}$ & $\begin{array}{c}\text { 4 months after the } \\
\text { manipulation }\end{array}$ \\
\hline Flexion & $102.5^{\circ}{ }_{(7.94)}^{\circ}$ & $152.2^{\circ}(4.57)$ & $157.5^{\circ}(5.53)$ \\
\hline Abduction & $83.8^{\mathrm{o}}{ }_{(7.05)}$ & $143.4^{\mathrm{o}}{ }_{(5.48)}$ & $150.7^{\mathrm{o}}(3.89)$ \\
\hline Ext. Rotation & $27.1^{\mathrm{o}}(3.83)$ & $45.4^{\circ}(5.08)$ & $52.1^{\circ}(4.3)$ \\
\hline Int. Rotation & $2.5_{(0.5)}$ & $4.1_{(0.7)}$ & $7.5_{(0.96)}$ \\
\hline
\end{tabular}

The values given in brackets are Standard deviations

Table 3: Comparison of the quick disabilities of the arm, shoulder and hand score before and after the manipulation of shoulder

\begin{tabular}{|l|c|}
\hline \multicolumn{1}{|c|}{ Condition of shoulder } & Quick DASH Score \\
\hline Before the shoulder manipulation & $83.7(7.15)$ \\
\hline After the shoulder manipulation & $27.6(7.04)$ \\
\hline \multicolumn{2}{|l|}{ Values given in brackets are standard deviation. P value $=0.0005$}
\end{tabular}

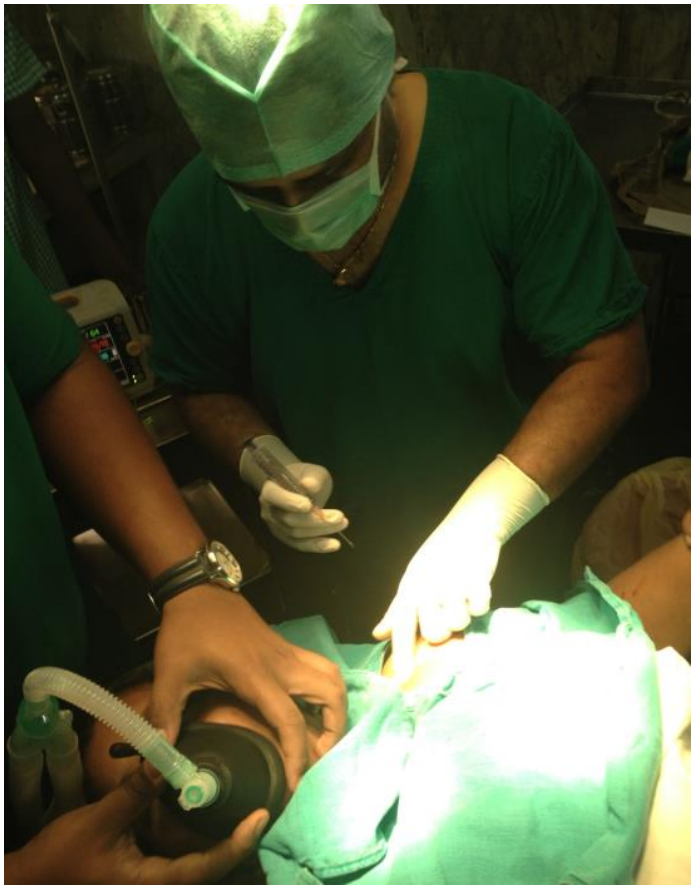

Fig. 1: Picture showing procedure for injecting cold normal saline in to shoulder joint (palpating coracoid process and injecting needle lateral to it)

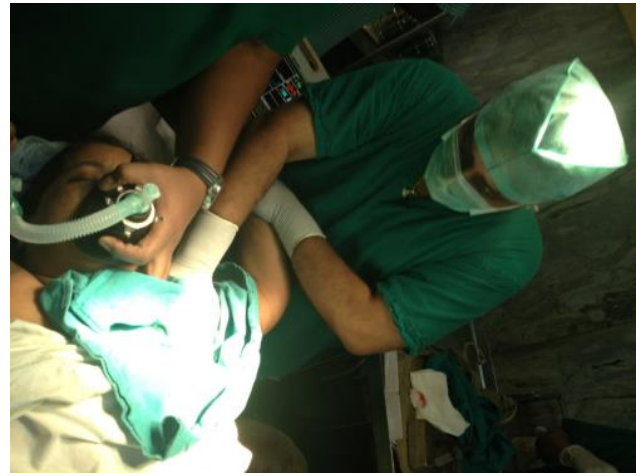

Fig. 2: Overhead abduction of shoulder after injection of cold normal saline and $2 \%$ xylocaine into the shoulder

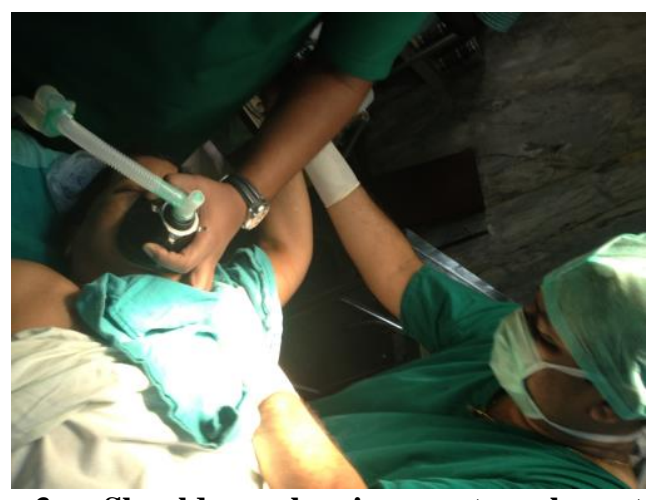

Fig. 3: Shoulder showing external rotation movement after over head abduction of shoulder for breaking of intra-articular adhesions 


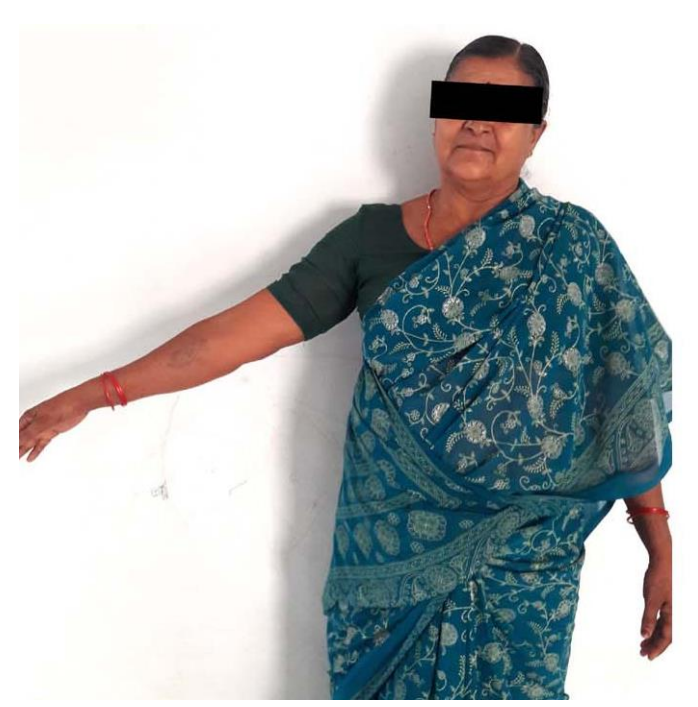

Fig. 4: Abduction before MUA

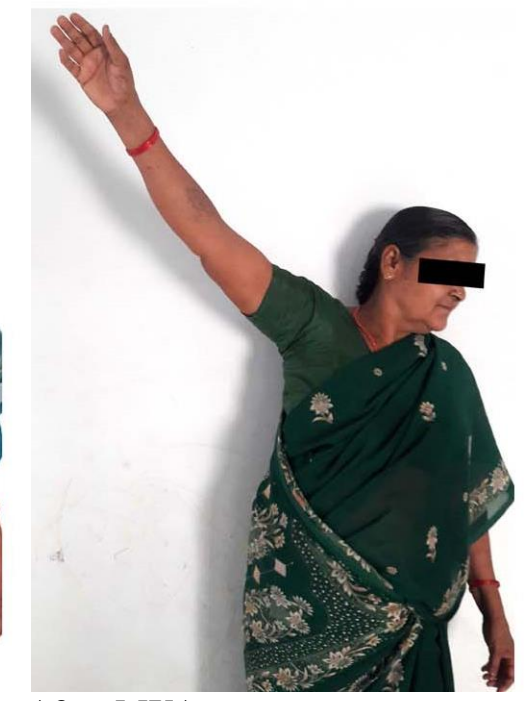

After MUA

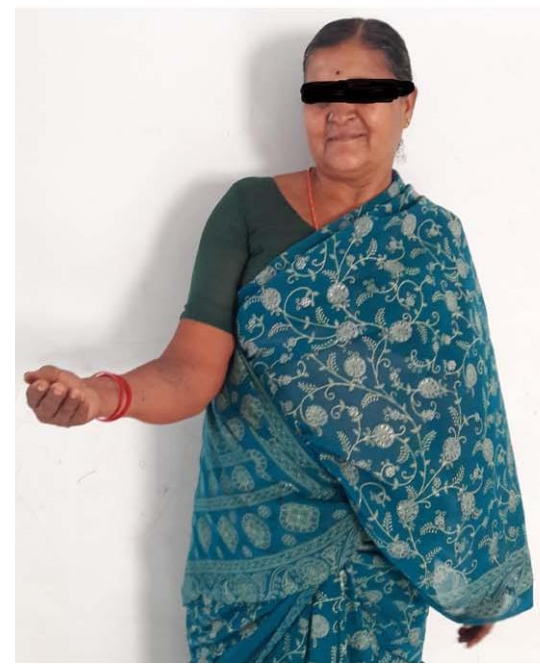

Fig. 5: External rotation before MUA

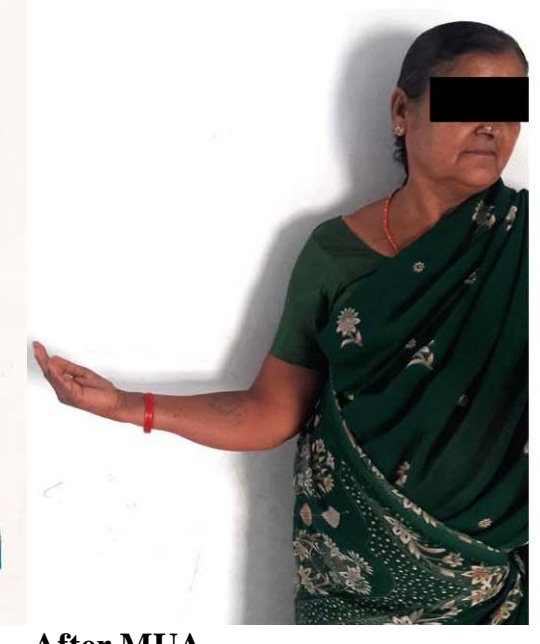

After MUA
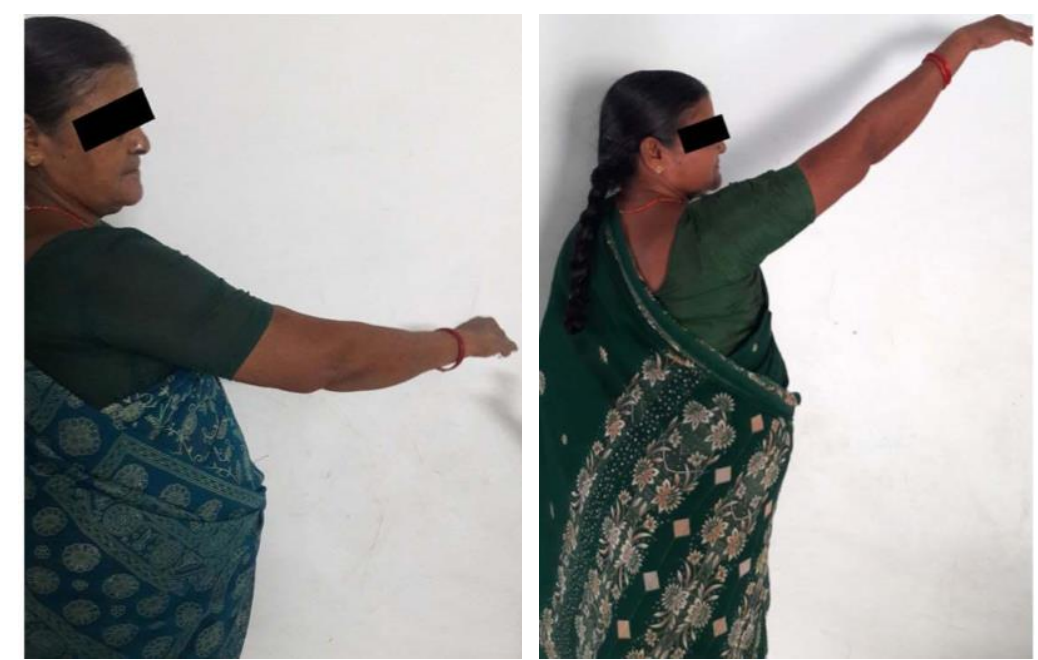

Fig. 6: Flexion before MUA

After MUA 


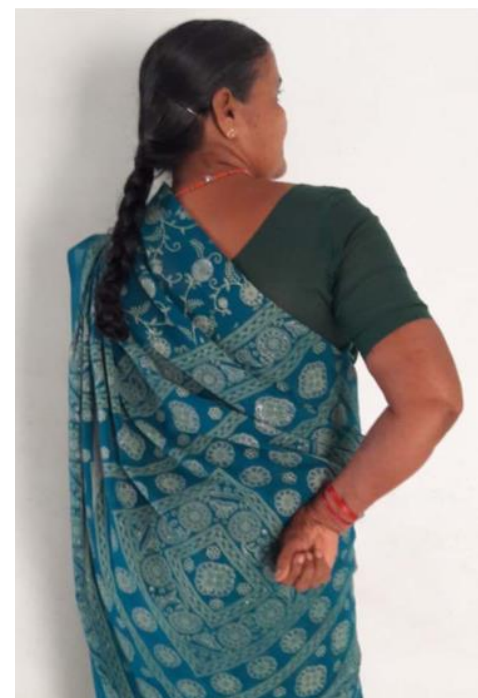

Fig. 7: Internal rotation before MUA

\section{Discussion}

Exact definition in natural history of frozen shoulder has traditionally been regarded as "a selflimiting condition, which universally settles over a variable time course." 4 This self-limiting condition of frozen shoulder was not accepted by many. Shaffer B et.al, in their study showed that "62 patients (68shoulders) followed-up over 7 years showed that $50 \%$ had either stiffness or mild pain and $60 \%$ had loss in shoulder Range of Motion mainly in the form of externarotation". 7

In this study, we have examined more than 100 patients with Periarthritis shoulder. These patients were started NSAIDs, universal shoulder mobilization exercises and physiotherapy for over 6 weeks. Around 60 patients showed improvement in shoulder pain and improvement in the range of motion. This shows that more than $59 \%$ of patient could be benefitted from physiotherapy, universal shoulder mobilization exercises, NSAIDs. In a study by Liaw SC et.al. Physiotherapy improves movements and function in frozen shoulder, (irrespective of onset of symptoms and delay in presentation to treatment). ${ }^{8}$ But earlier, the start of physiotherapy will be better recovery. ${ }^{8}$

In Kivimäki et al., (2007) study, observations showed no significant difference between two groups (MUA + Physiotherapy and Physiotherapy alone groups) in terms of pain relief, shoulder function and ROM over long term follow-up. ${ }^{12}$ Patients who could not show any improvement in range of motion, no decrease in shoulder pain after 6 weeks, with Oxford Shoulder score equal to or less than 19 were included in our study for the manipulation. Our observations hereafter show MUA with physiotherapy (immediate increase ROM, pain relief) shows quick recovery than physiotherapy (6 weeks for pain relief and increase in ROM) alone.

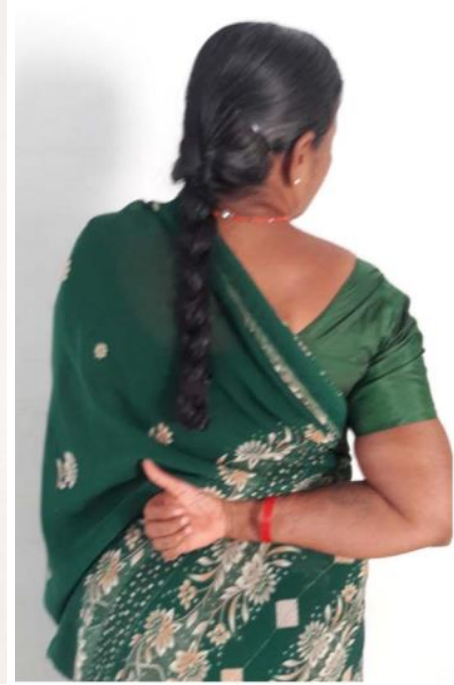

After MUA

In our study average age of patient was 54(39-65) years. Frozen shoulder normally occurs between the ages of 40-60years. In our study, there was sudden and abrupt increase in shoulder mobility immediately after procedure was observed. This could not be possible without any intervention. Injection of methylprednisolone $40 \mathrm{mg} / \mathrm{ml}(1 \mathrm{ml}), 2 \%$ lignocaine $5 \mathrm{cc}$ and $40 \mathrm{cc}$ of cold normal saline were injected intraarticularly into shoulder. $2 \%$ lignocaine injection provided immediate and good pain relief postoperatively. 40cc cold normal saline injection intraarticularly resulted in lysis of adhesions. Manipulation thereafter released the remaining adhesions. Postmanipulation shoulder range of movements improved immediately. This was shown by improvements in flexion $102.5^{\circ}( \pm 7.94)$ to $152.2^{\circ}( \pm 4.57)$, abduction $83.8^{0}( \pm 7.05)$ to $143.4^{0}( \pm 5.48)$, external rotation $27.1^{\circ}( \pm 3.83)$ to $45.4^{\circ}( \pm 5.08)$ and internal rotation $2.5( \pm 0.5)$ to $4.1( \pm 0.7)$. Universal shoulder mobilization exercises and physiotherapy were started immediately after MUA as patient regained conscious and being stable after procedure. Shoulder mobilization exercises started were continued for four months, which further showed increase in flexion $157.5^{\circ}( \pm 5.53)$, abduction $150.7^{0}( \pm 3.89)$, external rotation $52.1^{\circ}( \pm 4.3)$ and internal rotation $7.5( \pm 0.96)$.

In our study we evaluated VAS score of patients before and 4 months after the MUA shoulder. There was dramatic decrease in pain levels from 7.29(S.D \pm $1.03)$ to $1.71($ S.D. \pm 0.84$)(\mathrm{p}=0.0005)$. We could not record VAS score immediately after MUA because there was decrease in pain due to Post-Anesthesia and intra-articular Lignocaine injection. The Quick DASH scores values showing difficulty in carrying daily routine activities such as household work, recreational activities were improved. They could continue with daily activities without any difficulty. Mean of Quick DASH score in our study came down from 83 to 22 . 
Overall, the results of current study support MUA as an effective and safe treatment of the frozen shoulder. Complications such as fracture of proximal humerus ${ }^{9}$ (mainly over surgical neck area), brachial plexus injury, ${ }^{11}$ shoulder dislocation, ${ }^{9}$ post manipulation pain, ${ }^{9}$ hemarthrosis, tearing of the joint capsule ${ }^{10}$ or rotator cuff injury ${ }^{10}$ occur during MUA. In our study we did not experience any complication of MUA shoulder.

A small group of patients had no restricted movement during MUA. They needed no additional force for manipulation, showing movements restricted due to pain. This shows difficulty in diagnosis of adhesive capsulitis in normal clinical setting. Codman's observation in 1934 of frozen shoulder being a condition "difficult to define, difficult to treat and difficult to explain from the point of view of pathology" remains relevant today. ${ }^{1}$

In the end this study concludes that manipulation resulted in return of movements, but maintenance of movements over longer periods requires continuous shoulder physiotherapy and shoulder mobilization exercises. ${ }^{9,15}$

\section{Conclusion}

MUA followed by immediate physiotherapy, decreased shoulder pain and aided in good recovery of shoulder function in persons with Periarthritis shoulder. Intra-articular (Steriod + Lignocaine) and Cold Normal Saline during the procedure will result in Stretching of joint capsule, breaking of adhesions and good postoperative pain relief. This could help in start of physiotherapy at the earliest. Further MUA will result in breaking of remaining adhesions. MUA will result in return of movements. But without physiotherapy and continuous shoulder mobilization exercises, there will regression of movements of shoulder and result return of frozen shoulder symptoms.

\section{References}

1. Codman EA. The shoulder: Boston Thomas and Todd Co. 1934.

2. John Charnley, Periarthritis of the shoulder. Postgrad Med J. 1959;384-88.

3. Dodenhoff RM, Levy O, Wilson A. Manipulation under anesthesia for primary frozen shoulder: effect on early recovery and return to activity. J Shoulder Elbow Surg. 2000;9:23-26.

4. Reeves B. The natural history of frozen shoulder syndrome. Scand J Rheumatology. 1975;4(4):193196.

5. Bridgeman, J.F. Periarthritis of the Shoulder and Diabetes. Ann Rheum Dis. 1972;31:P39.

6. Halcuno A, Sashika H, Ohkawa T, Itoh R, Arthrographic findings in hemiplegic shoulders. Arch Phys Med Rehabil. 1984;65:706-11.

7. Shaffer B, Tibone JE, Kerlan RK. Frozen shoulder. A long-term followup. J Bone Joint Surg [Am]. 1992;74:738-746.

8. Liaw SC. The effect and timing of physiotherapy on change in range of motion and function in frozen shoulder. Physiother Singapore. 2000;3(3):82-86.
9. Reichmister. JP, friedman SL. Long term functional results after manipulation of frozen shoulder. Med $J$. 1999;48(1):7-11.

10. Ehud Atoun, Lennard Funk, Stephen A. Copland, Tirtza Even, Ofer Levy, Ehud Rath. The effect of shoulder manipulation on rotator cuff integrity. Acta Orthop Belg. 2013;79:255-259.

11. R Birch, J Jessop, G Scott. Brachial plexus palsy after manipulation of the shoulder. J Bone J Joint (Br). 1991;73-b:172.

12. Kivimaki J, Pohjolainen T, Malmivaara A. Manipulation under anesthesia with home exercises alone in the treatment of Frozen Shoulder: A randomized controlled trial with 125 patients. J Shoulder Elbow Surg. 2007;16(6):722-726.

13. Jacobs L.G, Smith MG, Khan SA, Smith K, Joshi M. Manipulation or intraarticular steroid in the management of adhesive capsulitis of shoulder? A prospective randomized trial. J Shoulder Elbow Surg. 2009; 18(B):348-53.

14. Quraoshi NA, Johmston P, Bayer J. Thawing the Frozen shoulder. A Randomized trial comparing manipulation under anesthesia with hydrodilation. J Bone Joint Surg Br. 2007;89 (9):1197-1200.

15. NgCy, Amin AK, Narborough S, McMallan L, Cook R, Brenkel JJ. Manipulation under anesthesia and early physiotherapy facilitate recovery in patients with frozen shoulder syndrome. Scott Med J. 2009;54(1):29-31.

How to cite this article: Avulapati S. K. Periarthritis shoulder - Effect of manipulation under general anesthesia on pain and range of movement. Indian $\mathbf{J}$ Orthop Surg. 2018;4(4):342-347. 